\title{
Squares in products with terms in an arithmetic progression
}

\author{
by
}

\author{
N. SARADHA (Mumbai)
}

1. Introduction. Let $d \geq 1, k \geq 2, l \geq 2, n \geq 1, y \geq 1$ be integers with $\operatorname{gcd}(n, d)=1$. Erdős [4] and Rigge [12] independently proved that a product of two or more consecutive positive integers is never a square. Further Erdős and Selfridge [5] showed that a product of $k$ consecutive integers is never a power, i.e.,

$$
n(n+1) \ldots(n+k-1)=y^{l} \text { with integers } k \geq 2, l \geq 2, n \geq 1, y \geq 1
$$

never holds. In [14, Corollary 1 ] the author extended this result by showing that

$$
n(n+d) \ldots(n+(k-1) d)=y^{l} \text { with integers } k \geq 3, l \geq 2, n \geq 1, y \geq 1
$$

never holds for $1<d \leq 6$. In this paper we extend the range of $d$ for the preceding equation with $l=2$.

THEOREM 1. The only solution of the equation

(1) $n(n+d) \ldots(n+(k-1) d)=y^{2} \quad$ in integers $k \geq 3, n \geq 1, y \geq 1$

and $1<d \leq 22$

is $(n, d, k)=(18,7,3)$.

Theorem 1 is a consequence of the following more general result.

TheOrem 2. Let $k \geq 3, n \geq 1$ and $1<d \leq 22$. Then there exists a prime exceeding $k$ which divides $n(n+d) \ldots(n+(k-1) d)$ to an odd power except when $(n, d, k) \in\{(2,7,3),(18,7,3),(64,17,3)\}$.

Equation (1) implies that every prime exceeding $k$ divides the product $n(n+d) \ldots(n+(k-1) d)$ to an even power. This contradicts Theorem 2 except when $(n, d, k) \in\{(2,7,3),(18,7,3),(64,17,3)\}$. But in these three cases we find that $n(n+d) \ldots(n+(k-1) d)$ equals $2 \cdot 12^{2}, 120^{2}, 2 \cdot 504^{2}$,

1991 Mathematics Subject Classification: Primary 11D61. 
respectively. Hence equation $(1)$ holds only when $(n, d, k)=(18,7,3)$. Thus Theorem 1 follows from Theorem 2. Let $P(m)$ denote the greatest prime factor of $m$ for any integer $m>1$ and we write $P(1)=1$. Then it follows from Theorem 2 that the equation

(2) $n(n+d) \ldots(n+(k-1) d)=B y^{2} \quad$ in positive integers $k \geq 3, n, y, B$

$$
\text { with } P(B) \leq k
$$

never holds for $1<d \leq 22$ except when $(n, d, k) \in\{(2,7,3),(18,7,3)$, $(64,17,3)\}$.

Marszałek [7] proved that equation (2) with $d>1$ and $B=1$ implies that

$$
k<2 \exp \left(d(d+1)^{1 / 2}\right) .
$$

Shorey and Tijdeman [16] proved that equation (2) with $d>1$ implies that

$$
k<d^{C / \log \log d}
$$

where $C$ is an effectively computable absolute constant. We prove

Theorem 3. Equation (2) with $d \geq 23$ implies that

$$
k< \begin{cases}4 d(\log d)^{2} & \text { if } d \text { is odd } \\ 1.3 d(\log d)^{2} & \text { if } d \text { is even. }\end{cases}
$$

In Theorem 3 we need to consider only $d \geq 23$ in view of Theorem 2 . The estimate (5) is a considerable improvement of (3). The estimate (4) involves an unspecified constant which turns out to be large. Therefore the estimate (5) is better than (4) for small values of $d$.

Now we exhibit infinitely many solutions in relatively prime integers $n \geq 1$ and $d>1$ of equation (2) with $k=3$ and square-free integer $B$ satisfying $P(B) \leq 3$. We observe that $B \in\{1,2,3,6\}$. For $B=1$, the existence of infinitely many solutions follows from a well known result that there are infinitely many triples of relatively prime squares in arithmetic progression. For $B>1$, we prove

Theorem 4. Let $B \in\{2,3,6\}$. There are infinitely many triples $(n, d, y)$ with integers $n \geq 1, d>1, y \geq 1$ and $\operatorname{gcd}(n, d)=1$ satisfying

$$
n(n+d)(n+2 d)=B y^{2} .
$$

Let $d=1, k \geq 3$ and $n(n+1) \ldots(n+k-1)$ be divisible by a prime greater than $k$. Then Erdös and Selfridge [5] proved that there exists a prime $p \geq k$ dividing $n(n+1) \ldots(n+k-1)$ to an odd power. The author [14] showed that the above assertion is valid with $p>k$ whenever $k \geq 4$. If $d=1$ and $k=3$, we prove

THEOREM 5. There is a prime exceeding 3 which divides $n(n+1)(n+2)$ to an odd power except when $n \in\{1,2,48\}$. 
When $n=1,2,48$, we see that $n(n+1)(n+2)$ equals $6,6 \cdot 2^{2}, 6 \cdot 140^{2}$ and the assertion of Theorem 5 is false. For the proof of Theorem 5 , it suffices to show that the equation

$$
n(n+1)(n+2)=B y^{2} \quad \text { with } B \in\{1,2,3,6\}
$$

has no solution other than $B=6,(n, y) \in\{(1,1),(2,2),(48,140)\}$. If $B=1$, the above assertion is a particular case of the result of Erdös and Rigge mentioned at the beginning of this section. If $B=6$ and $n$ odd, then the assertion was proved by Meyl [8] whereas Watson [17] and Ljunggren [6] proved the case of $n$ even.

The Algorithm in Section 3 was programmed and checkings and computations for the proof of Theorem 2 were carried out using Mathematica. I thank Professor T. N. Shorey for many helpful discussions. I also thank the referee for his valuable comments on an earlier draft of the paper.

2. Lemmas. We suppose throughout this section that $n \geq 1, d>1$ and $k \geq 3$ with $(n, d, k) \neq(2,7,3)$. Then by a result of Shorey and Tijdeman [15], we have

$$
P(n(n+d) \ldots(n+(k-1) d))>k .
$$

Further we suppose that

(9) $\operatorname{ord}_{p}(n(n+d) \ldots(n+(k-1) d)) \equiv 0(\bmod 2)$ for all primes $p>k$.

We write

(10) $n+i d=a_{i} x_{i}^{2}, \quad a_{i}$ square-free, $P\left(a_{i}\right) \leq k, x_{i}>0$ for $0 \leq i \leq k-1$.

We observe that $\operatorname{gcd}\left(a_{i}, d\right)=1$ for $0 \leq i \leq k-1 \operatorname{since} \operatorname{gcd}(n, d)=1$. We denote by $\left\{a_{1}^{\prime}, \ldots, a_{t^{\prime}}^{\prime}\right\}$ the set of all the distinct elements from $\left\{a_{0}, \ldots, a_{k-1}\right\}$. By (8), we have

$$
n+(k-1) d \geq(k+1)^{2} .
$$

Let $m \geq 1$ be an integer and $2 \leq p_{1}^{(d)}<p_{2}^{(d)}<\ldots$ be all the primes which are coprime to $d$. We define $B_{m, d}=\left\{a_{r}^{\prime} \mid P\left(a_{r}^{\prime}\right) \leq p_{m}^{(d)}\right\}$ and $g(k, m, d)=$ $\left|B_{m, d}\right|$. We observe that

$$
g(k, m, d) \geq t^{\prime}-\sum_{i \geq m+1}\left(\left[\frac{k}{p_{i}^{(d)}}\right]+\varepsilon_{i}^{(d)}\right):=g_{0}(k, m, d)
$$

where $\varepsilon_{i}^{(d)}=0$ if $p_{i}^{(d)}>k$ and for $p_{i}^{(d)} \leq k, \varepsilon_{i}^{(d)}=0$ or 1 according as $p_{i}^{(d)} \mid k$ or not for $i \geq m+1$. We note that $g(k, m, d)$ and $g_{0}(k, m, d)$ are the same as $g(k, m)$ and $g_{0}(k, m)$ of [14].

Throughout this section we assume without reference that $h$ is a positive integer with $h$ even whenever $d$ is even. Further, let $\varrho>0$. Define $V_{h}=$ $\left\{\alpha \mid \alpha\right.$ is a positive integer with $\alpha h^{2}<\varrho$ and $\left.\operatorname{gcd}(\alpha, d)=1\right\}$. We write 
$V_{h}=\bigcup_{i \geq 1} V_{h i}$ such that for every $i \geq 1$, positive integers $\alpha$ and $\beta$ are in $V_{h i}$ if and only if $\alpha \equiv \beta\left(\bmod \frac{d}{\varepsilon_{h} \operatorname{gcd}(d, h)}\right)$ where

$$
\varepsilon_{h}=1 \text { if } 2 \nmid \frac{d}{\operatorname{gcd}(d, h)} \quad \text { and } \quad \varepsilon_{h}=2 \text { if } 2 \mid \frac{d}{\operatorname{gcd}(d, h)} .
$$

Further, let $\delta_{h}=\max \left\{\left|V_{h i}\right|\right\}$ and $\delta(d)=\sum_{h<\sqrt{\varrho}}^{*} \delta_{h}$. Here we recall that the summation in $\sum^{*}$ is taken over even values of $h$ whenever $d$ is even. We note that $\delta(d)$ can be computed for every $d$ and $\varrho$ and that the values of $\delta(d)$ for $7 \leq d \leq 22$ and $\varrho=\frac{1}{3} d^{2}$ can be found in Table 1 . We begin with the following lemma which gives a lower bound for the number of distinct $a_{i}$, viz., $t^{\prime}$.

Lemma 1. Let $n \geq(k-1)^{2} d^{2} /(4 \varrho)$. If (9) holds, then $t^{\prime} \geq k-\delta(d)$.

Proof. Let $b_{1}, \ldots, b_{r}, \ldots$ be the $a_{j}$ 's which occur more than once with $n+i_{r} d=b_{r} x_{i_{r}}^{2}$ for $r \geq 1$ and such that $x_{i_{r}}$ is minimal, i.e., if $a_{i}=b_{r}$ with $i \neq i_{r}$, then $x_{i}>x_{i_{r}}$. For any $b_{r}$ with $r \geq 1$, we say that $b_{r}$ is repeated at the hth place if there exists some $j, 0 \leq j \leq k-1$, such that $a_{j}=b_{r}, j \neq i_{r}$, $x_{j}=x_{i_{r}}+h$ with $h \geq 1$. We observe that $j$ is uniquely determined. We set $W_{h}=\left\{a_{j} \mid 0 \leq j \leq k-1, a_{j}=b_{r}, j \neq i_{r}\right.$ and $x_{j}=x_{i_{r}}+h$ for some $\left.r\right\}$. In order to get a lower bound for the number of distinct $a_{j}$ 's, we need to get an upper bound for $\sum_{h>1}\left|W_{h}\right|$. We observe that $\left|W_{h}\right|$ is equal to the number of $b_{r}$ which are repeated at the $h$ th place. We proceed to find an upper bound for this number.

Suppose $b_{r}$ is repeated at the $h$ th place. Then by its definition, we obtain for some $j, 0 \leq j \leq k-1, j \neq i_{r}$,

$$
\begin{aligned}
(k-1) d & \geq\left(j-i_{r}\right) d=b_{r}\left(x_{j}^{2}-x_{i_{r}}^{2}\right)=b_{r}\left(2 h x_{i_{r}}+h^{2}\right) \\
& >2 h b_{r}^{1 / 2}\left(b_{r} x_{i_{r}}^{2}\right)^{1 / 2} \geq 2 h b_{r}^{1 / 2} n^{1 / 2}>h b_{r}^{1 / 2} \frac{(k-1) d}{\sqrt{\varrho}} .
\end{aligned}
$$

Thus

$$
b_{r} h^{2}<\varrho .
$$

Hence $h<\sqrt{\varrho}$, i.e., the number of places at which $b_{r}$ can be repeated is at most $[\sqrt{\varrho}]$. Further, we note from $(14),(10)$ and $(15)$ that $h$ is even whenever $d$ is even and that $b_{r} \in V_{h}$. Also we observe that $h\left(2 x_{i_{r}}+h\right) \equiv 0(\bmod d)$ from which it follows that $x_{i_{r}} \equiv c\left(\bmod \frac{d}{\varepsilon_{h} \operatorname{gcd}(d, h)}\right)$ where $c$ depends only on $h$ and $d$ with $\varepsilon_{h}$ as in (13). Thus $n \equiv b_{r} c^{2}\left(\bmod \frac{d}{\varepsilon_{h} \operatorname{gcd}(d, h)}\right)$. Further, we observe that $\operatorname{gcd}\left(c, \frac{d}{\varepsilon_{h} \operatorname{gcd}(d, h)}\right)=1$ since $\operatorname{gcd}(n, d)=1$.

If $b_{s} \neq b_{r}$ is such that $b_{s}$ is repeated at the $h$ th place, then by the foregoing argument, we have $b_{s} h^{2}<\varrho$ and $n \equiv b_{s} c^{2}\left(\bmod \frac{d}{\varepsilon_{h} \operatorname{gcd}(d, h)}\right)$. Thus $b_{r} \equiv b_{s}\left(\bmod \frac{d}{\varepsilon_{h} \operatorname{gcd}(d, h)}\right) \operatorname{since} \operatorname{gcd}\left(c, \frac{d}{\varepsilon_{h} \operatorname{gcd}(d, h)}\right)=1$. Hence $b_{r}, b_{s}$ belong to 
$V_{h i}$ for some $i$. Thus the number of $b_{r}$ which are repeated at the $h$ th place is $\leq \delta_{h}$. Since $h<\sqrt{\varrho}$, we have

$$
\sum_{h \geq 1}\left|W_{h}\right| \leq \sum_{h<\sqrt{\varrho}} \delta_{h}=\delta(d) .
$$

Hence the number of distinct $a_{j}$ 's is at least $k-\delta(d)$.

As a consequence of Lemma 1, we have

Corollary 1. Let $k \geq 2(2 d-7)$. If (9) holds, then $t^{\prime} \geq k-\delta(d)$ where $\delta(d)$ is computed with $\varrho=\frac{1}{3} d^{2}$.

Proof. By (11) and $k \geq 2(2 d-7)$, we see that

$$
n \geq(k+1)^{2}-(k-1) d>(k+1)^{2}-\frac{(k-1)(k+14)}{4}>\frac{3}{4}(k-1)^{2} .
$$

Now the result follows immediately from Lemma 1.

Let $1=s_{1}<s_{2}<\ldots$ be the sequence of all square-free integers and $1=s_{1}^{\prime}<s_{2}^{\prime}<\ldots$ be the sequence of all odd square-free integers.

LEMma 2. We have

(i) $s_{i} \geq(1.5) i$ for $i \geq 39$.

(ii) $s_{i} \geq(1.6) i$ for $286 \leq i \leq 570$.

(iii) $s_{i}^{\prime} \geq(2.25) i$ for $i \geq 12$.

Proof. (i) We first check that $s_{i} \geq(1.5) i$ for $39 \leq i \leq 70$. Further, we check that for $0 \leq r<36, r \notin S_{0}=\{0,4,8,9,12,16,18,20,24,27,28,32\}$ we can choose an $s_{i_{r}}$ with $39 \leq i_{r} \leq 70$ such that $s_{i_{r}} \equiv r(\bmod 36)$. Now we consider any $s_{i}$ with $i>70$. Then $s_{i} \equiv r(\bmod 36)$ for some $r$ with $0 \leq r<36, r \notin S_{0}$. Thus $s_{i} \equiv s_{i_{r}}(\bmod 36)$ with $39 \leq i_{r} \leq 70$. Hence

$$
s_{i}-s_{i_{r}}=36 f
$$

for some positive integer $f$. We know that in any set of 36 consecutive integers, the number of square-free integers is $\leq 24$. Thus the number of square-free integers $\leq 36 f$ is at most $24 f$. Also we observe from (16) that this number is equal to $i-i_{r}$. Therefore $i-i_{r} \leq 24 f \leq \frac{2}{3}\left(s_{i}-s_{i_{r}}\right)$ by (16). Hence $s_{i} \geq \frac{3}{2}\left(i-i_{r}\right)+s_{i_{r}} \geq(1.5) i$ since $s_{i_{r}} \geq(1.5) i_{r}$.

(ii) The inequality follows by direct checking.

(iii) We check that $s_{i}^{\prime} \geq(2.25) i$ for $12 \leq i \leq 35$. Also we check for $0 \leq r<36$ with $r \equiv 1(\bmod 2)$ and $r \notin S_{0}$ that we can choose an $s_{i_{r}}^{\prime}$ with $12 \leq i_{r} \leq 35$ such that $s_{i_{r}}^{\prime} \equiv r(\bmod 36)$. Further, we observe that the number of odd square-free integers in any set of 36 consecutive integers is $\leq 16$. Now we repeat the argument in (i) for any $s_{i}^{\prime}$ with $i>35$ to obtain (iii). 
It can be checked that

$\prod_{i=1}^{63} s_{i} \geq(1.5)^{63}(63) !, \quad \prod_{i=1}^{286} s_{i} \geq(1.6)^{286}(286) !, \quad \prod_{i=1}^{51} s_{i}^{\prime} \geq(2.25)^{51}(51) !$.

By Lemma 2 and from an induction argument we derive

COROllary 2. We have

(i) $\prod_{i=1}^{\nu} s_{i} \geq(1.5)^{\nu} \nu$ ! for $\nu \geq 63$.

(ii) $\prod_{i=1}^{\nu} s_{i} \geq(1.6)^{\nu} \nu$ ! for $286 \leq \nu \leq 570$.

(iii) $\prod_{i=1}^{\nu} s_{i}^{\prime} \geq(2.25)^{\nu} \nu$ ! for $\nu \geq 51$.

The inequality in (i) of the above corollary has already appeared in [5].

Lemma 3. Let $7 \leq d \leq 22$. Suppose (9) holds. Then $k \leq k_{0}(d):=k_{0}$ where $k_{0}$ is as given in Table 1 .

Pr o o f. Suppose $k>k_{0}$. Then $k>2(2 d-7)$. Hence Corollary 1 is valid. Thus $t^{\prime} \geq k-\delta(d)$ where $\delta(d)$ is computed with $\varrho=\frac{1}{3} d^{2}$. We note from Table 1 that $\delta(d) \leq 20$. Thus $t^{\prime} \geq k-20$. From now onwards we shall assume that $k \geq 83$. Since $a_{i}^{\prime}$ for $1 \leq i \leq t^{\prime}$ are square-free integers, we use Corollary 2(i) to obtain

$$
\prod_{i=1}^{t^{\prime}} a_{i}^{\prime} \geq \prod_{i=1}^{k-20} a_{i}^{\prime} \geq \prod_{i=1}^{k-20} s_{i} \geq(1.5)^{k-20}(k-20) !
$$

On the other hand, by (10), we have

$$
a_{1}^{\prime} \ldots a_{t^{\prime}}^{\prime} \mid(k-1) ! \prod_{p \leq k} p .
$$

We put $g_{q}=\operatorname{ord}_{q}\left(a_{1}^{\prime} \ldots a_{t^{\prime}}^{\prime}\right)$ and $h_{q}=\operatorname{ord}_{q}\left((k-1) ! \prod_{p \leq k} p\right)$ for any prime $q \leq k$. Then it follows from Marszałek [7, p. 221] that

$$
g_{q} \leq \frac{k}{q+1}+\frac{\log k}{\log q}+1 \quad \text { and } \quad h_{q} \geq \frac{k-1}{q-1}-\frac{\log k}{\log q}
$$

Thus

$$
g_{q}-h_{q} \leq \frac{-2 k}{q^{2}-1}+\frac{q}{q-1}+\frac{2 \log k}{\log q} .
$$

Further, from (18) we get

$$
a_{1}^{\prime} \ldots a_{t^{\prime}}^{\prime} \mid(k-1) !\left(\prod_{p \leq k} p\right)\left(\prod_{q \leq 19} q^{g_{q}-h_{q}}\right)
$$

where in the product signs $p, q$ run over primes. Now by (20) and (19), we have

$$
a_{1}^{\prime} \ldots a_{t^{\prime}}^{\prime} \leq(k-1) !\left(\prod_{p \leq k} p\right) k^{16}\left(\prod_{q \leq 19} q^{q /(q-1)}\right)\left(\prod_{q \leq 19} q^{2 /\left(q^{2}-1\right)}\right)^{-k} .
$$


We find that

$$
\left\{\begin{array}{l}
\prod_{q \leq 19} q^{q /(q-1)} \leq 153819970, \quad \prod_{q \leq 19} q^{2 /\left(q^{2}-1\right)} \geq 2.8819, \\
\prod_{p \leq k} p \leq(2.78)^{k} \quad(\text { see }[13, \text { p. } 71]) .
\end{array}\right.
$$

Using (22) in (21) and comparing with (17), we get

$$
(1.5549)^{k} \leq(153819970)(1.5)^{20} k^{35} .
$$

This inequality is not valid for $k \geq 570$. Thus we obtain $k<570$. Now let $k \geq 485$. We use Corollary 2(ii) to get

$$
\prod_{i=1}^{t^{\prime}} a_{i}^{\prime} \geq(1.6)^{k-20}(k-20) ! .
$$

Comparing this lower bound with the upper bound in (21), we get

$$
(1.6586)^{k} \leq(153819970)(1.6)^{20} k^{35} \text {. }
$$

This inequality is not valid for $k \geq 485$. Thus we conclude that $k<485$.

We shall bring down the value of $k$ to $k_{0}$ in all cases except $d=19$ by a counting argument which will be presented in the next paragraph. When $d=19$ the counting argument fails. But a refinement of the above argument itself enables us to bring $k<315$. When $d=19$ we observe that $g_{19}=0$ and we rewrite (20) as

$$
a_{1}^{\prime} \ldots a_{t^{\prime}}^{\prime} \leq(k-1) !\left(\prod_{p \leq k} p\right)\left(\prod_{q \leq 17} q^{g_{q}-h_{q}}\right)(19)^{-h_{q}} .
$$

On the other hand, by Corollary 2(ii), we have for $485>k \geq 303, a_{1}^{\prime} \ldots a_{t^{\prime}}^{\prime} \geq$ $(1.6)^{k-17}(k-17)$ ! since $\delta(d)=17$. Now we combine the preceding estimates for $a_{1}^{\prime} \ldots a_{t^{\prime}}^{\prime}$ to conclude that $k<315$ whenever $d=19$.

Let $7 \leq d \leq 22, d \neq 19$ and $k<485$. Since $a_{i}^{\prime}$ for $1 \leq i \leq t^{\prime}$ are distinct and square-free we have

$$
g(k, m, d) \leq 1+\left(\begin{array}{c}
m \\
1
\end{array}\right)+\ldots+\left(\begin{array}{c}
m \\
m
\end{array}\right)=2^{m} .
$$

Thus if $g_{0}(k, m, d) \geq 2^{m}+1$, we get a contradiction by (12). Since $t^{\prime} \geq$ $k-\delta(d)$, we replace $t^{\prime}$ in (12) by $k-\delta(d)$ and using Table 1 for the values of $\delta(d)$ we check that $g_{0}(k, m, d) \geq 2^{m}+1$ for a proper choice of $m$ whenever $k_{0}<k<485$. For example, when $d=13$ we observe from $p_{6}^{(d)}=17$ and the definition of $g_{0}(k, m, d)$ that $g_{0}(k, 5,13) \geq 33$ for $120 \leq k<485$. The other cases are checked similarly. See Table 1 for the choices of $m$ when different values of $d$ and $k$ are considered. This completes the proof. 
Table 1

\begin{tabular}{ccccc|ccccc}
\hline$d$ & $\delta(d)$ & Range for $k$ & $m$ & $k_{0}$ & $d$ & $\delta(d)$ & Range for $k$ & $m$ & $k_{0}$ \\
\hline 7 & 6 & $27-310$ & 3 & 26 & 14 & 5 & $43-349$ & 2 & 42 \\
& & $311-484$ & 4 & & & & $350-484$ & 3 & \\
8 & 4 & $19-20$ & 2 & 18 & 15 & 14 & $47-484$ & 3 & 46 \\
& & $21-106$ & 3 & & 16 & 11 & $51-238$ & 3 & 50 \\
& & $107-318$ & 4 & & & & $239-484$ & 4 & \\
& & $319-484$ & 5 & & 17 & 15 & $255-484$ & 6 & 254 \\
9 & 7 & $40-285$ & 3 & 39 & 18 & 6 & $59-102$ & 1 & 58 \\
& & $286-484$ & 4 & & & & $103-348$ & 2 & \\
10 & 2 & $27-136$ & 1 & 28 & & & $349-484$ & 3 & \\
& & $137-383$ & 2 & & 19 & 17 & - & - & 314 \\
& & $384-484$ & 3 & & 20 & 8 & $67-318$ & 2 & 66 \\
11 & 9 & $55-484$ & 4 & 54 & & & $319-484$ & 3 & \\
12 & 4 & $35-372$ & 2 & 34 & 21 & 20 & $100-484$ & 3 & 99 \\
& & $373-484$ & 3 & & 22 & 7 & $75-310$ & 3 & 74 \\
13 & 12 & $120-484$ & 5 & 119 & & & $311-484$ & 4 & \\
\hline
\end{tabular}

We see from Table 1 that $\delta(d) \leq 20$ for $7 \leq d \leq 22$. In the following lemma we give an upper bound for $\delta(d)$ whenever $d \geq 23$.

Lemma 4. For $d \geq 23$ and $\varrho=\frac{1}{3} d^{2}$, we have

$$
\delta(d) \leq \begin{cases}\frac{1}{4} d \log d+(.8323) d & \text { if } d \text { is odd } \\ \frac{1}{3} d \log d+(.118) d & \text { if } d \text { is even } .\end{cases}
$$

Proof. By the definition of $\delta(d)$, we obtain

$$
\delta(d) \leq \sum_{h<d / \sqrt{3}}\left(\left[\frac{d^{2}}{3 h^{2}} \cdot \frac{\varepsilon_{h} \operatorname{gcd}(d, h)}{d}\right]+1\right)
$$

where the sum is taken over even values of $h$ whenever $d$ is even. We observe from (13) that $\varepsilon_{h} \leq 2$. Thus from (24) we get

$$
\delta(d) \leq \frac{d}{3} \sum_{h<d /(2 \sqrt{3})} \frac{1}{h}+\frac{d}{2 \sqrt{3}} \quad \text { if } d \text { is even. }
$$

Let $d$ be odd. Then by (13), $\varepsilon_{h}=1$. Further, $\operatorname{gcd}(d, h) \leq h / 2$ whenever $h$ is even. Hence from (24) we get

$$
\begin{aligned}
\delta(d) & \leq \frac{d}{3} \sum_{h<d / \sqrt{3}, h \text { odd }} \frac{1}{h}+\frac{d}{3} \sum_{h<d / \sqrt{3}, h \text { even }} \frac{1}{2 h}+\frac{d}{\sqrt{3}} \\
& \leq \frac{d}{3} \sum_{h<d / \sqrt{3}} \frac{1}{h}-\frac{d}{6} \sum_{h<d / \sqrt{3}, h \text { even }} \frac{1}{h}+\frac{d}{\sqrt{3}}
\end{aligned}
$$




$$
\begin{aligned}
& \leq \frac{d}{3} \sum_{h<d / \sqrt{3}} \frac{1}{h}-\frac{d}{12} \sum_{h<d /(2 \sqrt{3})} \frac{1}{h}+\frac{d}{\sqrt{3}} \\
& \leq \frac{d}{4} \sum_{h<d /(2 \sqrt{3})} \frac{1}{h}+\frac{d}{3} \sum_{d /(2 \sqrt{3})<h<d / \sqrt{3}} \frac{1}{h}+\frac{d}{\sqrt{3}} .
\end{aligned}
$$

Thus

$$
\delta(d) \leq \frac{d}{4} \sum_{h<d /(2 \sqrt{3})} \frac{1}{h}+\frac{d}{3}+\frac{d}{\sqrt{3}}+\frac{2}{\sqrt{3}} \quad \text { if } d \text { is odd }
$$

We use $\sum_{h<x} 1 / h<\log x+\gamma+1 / x$ where $x>1$ and $\gamma$ is Euler's constant whose value is $<.5773$ (see [1, p. 55] and [13, pp. 65-66]) and $d \geq 23$ in the estimates (25) and (26) to prove the assertion of the lemma.

Lemma 5. Let $k=3$. Suppose (9) holds. Then $\left(a_{0}, a_{1}, a_{2}\right) \in S$ where $S=S_{1} \cup S_{2} \cup S_{3} \cup S_{4}$ with $S_{1}=\{(1,1,1)\}, S_{2}=\{(2,1,2)\}, S_{3}=\{(1,2,3)$, $(2,3,1),(3,1,2),(6,1,2),(1,1,2)\}$ and $S_{4}=\{(1,3,2),(2,1,3),(3,2,1)$, $(2,1,6),(2,1,1)\}$. Further, we have

$$
d \equiv \begin{cases}0(\bmod 8) & \text { if }\left(a_{0}, a_{1}, a_{2}\right) \in S_{1} \\ \pm 1(\bmod 8) & \text { if }\left(a_{0}, a_{1}, a_{2}\right) \in S_{2} \\ 1(\bmod 8) & \text { if }\left(a_{0}, a_{1}, a_{2}\right) \in S_{3} \\ -1(\bmod 8) & \text { if }\left(a_{0}, a_{1}, a_{2}\right) \in S_{4}\end{cases}
$$

Proof. From (9), we see that (10) holds and therefore $\left\{a_{0}, a_{1}, a_{2}\right\} \subset$ $\{1,2,3,6\}$. Also $\operatorname{gcd}(n, n+d)=\operatorname{gcd}(n+d, n+2 d)=1$ and $\operatorname{gcd}(n, n+2 d)=1$ or 2 since $\operatorname{gcd}(n, d)=1$. Thus we find that there are 20 possible values for the triple $\left(a_{0}, a_{1}, a_{2}\right)$, viz., $(1,1,1),(1,1,2),(1,1,3),(1,1,6),(1,2,1),(1,2,3)$, $(1,3,1),(1,3,2),(1,6,1),(2,1,1),(2,1,2),(2,1,3),(2,1,6),(2,3,1),(2,3,2)$, $(3,1,1),(3,1,2),(3,2,1),(6,1,1),(6,1,2)$.

We use without mentioning that $x_{0}, x_{1}, x_{2}$ are pairwise coprime and $a_{0} x_{0}^{2}+a_{2} x_{2}^{2}=2 a_{1} x_{1}^{2}$. We exclude the possibilities $(1,1,3)$ and $(1,1,6)$ since $2 x_{1}^{2}-x_{0}^{2} \not \equiv 0(\bmod 3) ;(1,3,1),(1,6,1),(2,3,2)$ since $x_{0}^{2}+x_{2}^{2} \not \equiv 0(\bmod 3)$; $(3,1,1)$ and $(6,1,1)$ since $2 x_{1}^{2}-x_{2}^{2} \not \equiv 0(\bmod 3) ;(1,2,1)$ since $x_{0}^{2}+x_{2}^{2} \not \equiv 0$ $(\bmod 4)$. The remaining 12 possibilities are given by $S$.

We take $\left(a_{0}, a_{1}, a_{2}\right)=(1,1,1) \in S_{1}$. Then $n=x_{0}^{2}, n+d=x_{1}^{2}, n+2 d=x_{2}^{2}$, implying $x_{0}$ and $x_{2}$ are odd since $n$ and $n+2 d$ are both odd or both even and $\operatorname{gcd}(n, n+2 d)=1$ or 2 . Thus $2 d=x_{2}^{2}-x_{0}^{2} \equiv 0(\bmod 8)$, yielding $d \equiv 0,4(\bmod 8)$. If $d \equiv 4(\bmod 8)$, then $x_{1}^{2}=n+d \equiv 5(\bmod 8)$, which is not possible. Thus $d \equiv 0(\bmod 8)$.

Next we consider $\left(a_{0}, a_{1}, a_{2}\right)=(2,1,2) \in S_{2}$. Then $x_{1}$ is odd and $n \equiv 0$ or $2(\bmod 8)$ according as $x_{0}$ is even or odd. Hence $1 \equiv x_{1}^{2}=n+d \equiv d$ or $d+2(\bmod 8)$, which implies that $d \equiv 1$ or $7(\bmod 8)$. 
Now we take $\left(a_{0}, a_{1}, a_{2}\right)=(1,2,3) \in S_{3}$. Then $x_{0}, x_{2}$ are odd and $d=$ $2 x_{1}^{2}-x_{0}^{2} \equiv-1$ or $1(\bmod 8)$ according as $x_{1}$ is even or odd. If $d \equiv-1$ $(\bmod 8)$, then $d=3 x_{2}^{2}-2 x_{1}^{2} \equiv 3-0 \equiv 3(\bmod 8)$, a contradiction. Thus $d \equiv 1(\bmod 8)$. Similarly we prove for other possibilities in $S_{3}$ that $d \equiv$ $1(\bmod 8)$.

Lastly, we consider $\left(a_{0}, a_{1}, a_{2}\right)=(1,3,2) \in S_{4}$. Then $x_{0}$ is even and hence $x_{1}, x_{2}$ are odd. Thus $d=2 x_{2}^{2}-3 x_{1}^{2} \equiv 7(\bmod 8)$. Likewise we prove for other possibilities in $S_{4}$ that $d \equiv 7(\bmod 8)$.

Lemma 6. Let $k=3$. Suppose (9) holds. Then one of the following possibilities holds: (i) $d=1$, (ii) $d \geq 23$, (iii) $(n, d) \in\{(2,7),(18,7),(64,17)\}$.

Pr o o f. Let $1<d<23$. We shall show that (iii) holds. By Lemma 5 , we need to consider $\left(a_{0}, a_{1}, a_{2}\right) \in S_{1}$ with $d=8,16 ;\left(a_{0}, a_{1}, a_{2}\right) \in S_{2} \cup S_{3}$ with $d=9,17$ and $\left(a_{0}, a_{1}, a_{2}\right) \in S_{2} \cup S_{4}$ with $d=7,15$.

Let $\left(a_{0}, a_{1}, a_{2}\right) \in S_{1}$ with $d=8,16$. Since $x_{1}^{2}-x_{0}^{2}=d$ we find that $x_{0}=1, x_{1}=3$ and $x_{0}=3, x_{1}=5$ and hence $x_{2}^{2}=17$ and 41 , respectively. This is not possible.

Let $\left(a_{0}, a_{1}, a_{2}\right) \in S_{2}$ with $d=7,9,15,17$. Then $x_{2}^{2}-x_{0}^{2}=d$ implies that $(n, d)=(18,7)$.

Let $\left(a_{0}, a_{1}, a_{2}\right) \in S_{3}$ with $d=9,17$. Let $d=9$. In the first 4 possibilities in $S_{3}$ we observe that 3 divides one of $n, n+d, n+2 d$. Hence $3 \mid n$, which is a contradiction since $\operatorname{gcd}(n, d)=1$. Let $\left(a_{0}, a_{1}, a_{2}\right)=(1,1,2)$. Then $x_{1}^{2}-x_{0}^{2}=$ 9 gives $x_{0}=4$ and hence $n+2 d=2 x_{2}^{2}=34$, which is impossible. Let $d=17$ and $\left(a_{0}, a_{1}, a_{2}\right)=(1,2,3)$. Then $n+34 \equiv 0,3(\bmod 9)$, implying $x_{0}^{2}=n \equiv 2,5(\bmod 9)$, a contradiction. The next three possibilities are excluded similarly. Let $\left(a_{0}, a_{1}, a_{2}\right)=(1,1,2)$. Then $x_{1}^{2}-x_{0}^{2}=17$ implies that $\left(x_{0}, x_{1}, x_{2}\right)=(8,9,7)$. Thus $(n, d)=(64,17)$.

Let $\left(a_{0}, a_{1}, a_{2}\right) \in S_{4}$ with $d=7,15$. Let $d=7$ and $\left(a_{0}, a_{1}, a_{2}\right)=(1,3,2)$. Then $n+7 \equiv 0,3(\bmod 9)$. Hence $x_{0}^{2}=n \equiv 2,5(\bmod 9)$, a contradiction. The next three possibilities are excluded similarly. Let $\left(a_{0}, a_{1}, a_{2}\right)=(2,1,1)$. Then $x_{2}^{2}-x_{1}^{2}=7$ gives $\left(x_{0}, x_{1}, x_{2}\right)=(1,3,4)$. Thus $(n, d)=(2,7)$. Let $d=15$. The first four possibilities in $S_{4}$ are excluded since 3 divides one of $n, n+d, n+2 d$. Let $\left(a_{0}, a_{1}, a_{2}\right)=(2,1,1)$. Then $x_{2}^{2}-x_{1}^{2}=15$ implies that $x_{1}=7$ or 1 , giving $2 x_{0}^{2}=n=34$ or -14 , which are impossible.

Lemma 7 . Let $7 \leq d \leq 22$ and $(n, d) \notin\{(2,7),(18,7),(64,17)\}$. Assume that $t^{\prime}=k$. Then (9) does not hold.

Pr o of. Suppose (9) holds. Then by Lemmas 3,6 and Table 1, we have $4 \leq k \leq k_{0} \leq 314$. We observe that (10) holds and $a_{0}, \ldots, a_{k-1}$ are all distinct since $t^{\prime}=k$. We often use these facts and the property that 
$\operatorname{gcd}\left(a_{i}, d\right)=1$ for $0 \leq i<k$ without any reference. We check that

$$
\begin{cases}g_{0}(k, 1, d) \geq 3 & \text { for } 4 \leq k \leq 8 \text { if } 2 \text { or } 3 \text { divides } d \\ g_{0}(k, 2,7) \geq 5 & \text { for } k=7,8 \\ g_{0}(k, 2, d) \geq 5 & \text { for } 9 \leq k \leq 22 \\ g_{0}(k, 3, d) \geq 9 & \text { for } 23 \leq k \leq 78 \\ g_{0}(k, 4, d) \geq 17 & \text { for } 79 \leq k \leq 276 \\ g_{0}(k, 5, d) \geq 33 & \text { for } 277 \leq k \leq 314\end{cases}
$$

But (27) contradicts (23), by (12). Thus we may assume that $4 \leq k \leq 6$ if $d=7$ and $4 \leq k \leq 8$ if $d \in\{11,13,17,19\}$.

Let $k=4$ and $d \in\{7,11,13,17,19\}$. We know that $P\left(a_{i}\right) \leq 3$ and hence $a_{i} \in\{1,2,3,6\}$. Thus $n(n+d)(n+2 d)(n+3 d)$ is a square. But this is impossible by a well known result of Euler (see Dickson [3, p. 635] and Mordell [9, p. 21, Corollary]). We also use this fact without reference when we deal with other values of $k$.

Let $k=5$. Since $a_{i}$ 's are distinct, we need only consider the case when 5 divides one and only one of $n, n+d, n+2 d, n+3 d, n+4 d$ and hence at most one $a_{i}$. The values of the other $a_{i}$ 's belong to $\{1,2,3,6\}$. We may assume that 5 divides one of $n+d, n+2 d, n+3 d$. Suppose $5 \mid n+d$. Then $\{n, n+2 d, n+3 d, n+4 d\} \in\left\{y_{1}^{2}, 2 y_{2}^{2}, 3 y_{3}^{2}, 6 y_{4}^{2}\right\}$ for some positive integers $y_{1}, y_{2}, y_{3}, y_{4}$. We explain the case $d=7$. Then $n \equiv 3(\bmod 5)$. Hence $n=2 y_{2}^{2}$ or $3 y_{3}^{2}$. Let $n=2 y_{2}^{2}$. Then $n+14=3 y_{3}^{2}, n+21=y_{1}^{2}$ and hence $n+28=6 y_{4}^{2}$, which gives $3 \mid 14$, a contradiction. When $n=3 y_{3}^{2}$, we get $n+14=2 y_{2}^{2}$, $n+21=y_{1}^{2}$ and hence $n+28=6 y_{4}^{2}$, implying $3 \mid 28$, a contradiction. As another example, we take $d=11$. Then $n \equiv 4(\bmod 5)$. We find that $n=6 y_{4}^{2}, n+22=y_{1}^{2}, n+33=3 y_{3}^{2}, n+44=2 y_{2}^{2}$. Here we observe that $y_{1}$ is even, $y_{2}, y_{3}, y_{4}$ are odd. Hence $n \equiv 6(\bmod 8)$ and $n+33 \equiv 7(\bmod 8)$. But $n+33=3 y_{3}^{2} \equiv 3(\bmod 8)$, a contradiction. By a similar argument, we exclude all the cases $5|n+d, 5| n+2 d, 5 \mid n+3 d$ for $d \in\{7,11,13,17,19\}$. Thus $k \neq 5$.

Let $k=6$. Then $P\left(a_{i}\right) \leq 5$ and we may assume that $5 \nmid n$. Hence 5 divides only one of $\{n+d, n+2 d, n+3 d, n+4 d\}$. Therefore five of the $a_{i}$ 's belong to $\{1,2,3,6\}$. This is not possible since $a_{i}$ 's are all distinct. Thus $k \neq 6$.

Let $k=7$ and $d \in\{11,13,17,19\}$. Then $P\left(a_{i}\right) \leq 7$ and we may assume that there exist distinct $i_{1}, i_{2}$ and $i_{3}$ between 0 and 6 such that $7 \mid n+i_{1} d$, $5\left|n+i_{2} d, 5\right| n+i_{3} d$ since otherwise $g_{0}(k, 2, d) \geq 5$ leading to a contradiction. There are 8 possibilities for $\left(i_{1}, i_{2}, i_{3}\right)$ for each $d$. We check the case $7 \mid n+d$, $5|n, 5| n+5 d$ for $d=17$. Then $n+2 d=6 y_{4}^{2}, n+3 d=y_{1}^{2}, n+4 d=2 y_{2}^{2}$ and hence $n+6 d=3 y_{3}^{2}$, which implies $3 \mid 4 d$, a contradiction. The other cases are excluded similarly.

Finally, let $k=8$ and $d \in\{11,13,17,19\}$. Then $P\left(a_{i}\right) \leq 7$ and we may assume that $7|n, 7| n+7 d, 5|n+d, 5| n+6 d$ for otherwise $g_{0}(k, 2, d) \geq 5$, 
which is a contradiction. Then $(n+2 d)(n+3 d)(n+4 d)(n+5 d)$ is a square, which is impossible.

The following lemma deals with the integral solutions of certain Diophantine equations.

LEMma 8. (i) There are infinitely many integral solutions in $x$ and $y$ of the equation $x^{2}-2 y^{2}=1$ with $x$ odd and of the equation $x^{2}-3 y^{2}=1$ with $x$ odd as well as with $x$ even.

(ii) All solutions of the equation $3 x^{2}+y^{2}=z^{2}$ in integers $x, y$, and $z$ are given by

$$
x=\varrho_{0} u s, \quad y=\frac{1}{2} \varrho_{0}\left(\alpha u^{2}-\beta s^{2}\right), \quad z=\frac{1}{2} \varrho_{0}\left(\alpha u^{2}+\beta s^{2}\right)
$$

where $\alpha \beta=3, u$ and $s$ are positive integers with $\operatorname{gcd}(u, s)=1$ and $\varrho_{0}$ is any integer when $u$ and $s$ are odd but $\varrho_{0}$ is even when one of $u$ and $s$ is even and the other is odd.

(iii) The only solutions in non-zero integers of $x^{4}+y^{4}=2 z^{2}$ with $\operatorname{gcd}(x, y)=1$ are $x^{2}=1, y^{2}=1$ and $z^{2}=1$. There is no solution in non-zero integers of the equation $x^{4}-y^{4}=2 z^{2}$ with $\operatorname{gcd}(x, y)=1$.

Lemma 8(i) is a well known result in continued fraction theory. We refer to $[10$, Theorem 7.25 , pp. 173-174] from where the result in Lemma 8(i) can be derived easily using the facts that $\sqrt{2}=\langle 1, \overline{2}\rangle$ and $\sqrt{3}=\langle 1, \overline{1,2}\rangle$. Lemma 8(ii) can be found in [2, pp. 40-41]. The first assertion in Lemma 8(iii) is proved in [11, p. 38]. It also follows from A14.4 of [11, p. 171]. In fact, the statement given therein should be corrected as: If $m \geq 0$ and $x^{4}+y^{4}=2^{m} z^{2}$ with $\operatorname{gcd}(x, y)=1$, then $m=1$ and $x^{2}=y^{2}=z^{2}=1$. The second assertion in Lemma 8(iii) follows from A14.5 of [11, p. 172].

3. An algorithm. In this section, we modify the algorithm given in $[14, \S 4]$.

Algorithm. Let $d$ and $k \geq 4$ be given. Also let $\mu>0$.

STEP 1. Find all primes $q_{1}, \ldots, q_{\theta}, q_{\theta+1}, \ldots, q_{\theta+\eta}$ which are coprime to $d$ and such that $q_{1}<\ldots<q_{\theta} \leq k<q_{\theta+1}<\ldots<q_{\theta+\eta}$ and $q_{i}^{2}<k^{2} d^{2} / \mu$ for $1 \leq i \leq \theta+\eta$.

STEP 2. Set $D=\left\{q_{1}^{\alpha_{1}} \ldots q_{\theta}^{\alpha_{\theta}} q_{\theta+1}^{2 \beta_{1}} \ldots q_{\theta+\eta}^{2 \beta_{\eta}} \mid q_{1}^{\alpha_{1}} \ldots q_{\theta}^{\alpha_{\theta}} q_{\theta+1}^{2 \beta_{1}} \ldots q_{\theta+\eta}^{2 \beta_{\eta}}\right.$ $<k^{2} d^{2} / \mu$ for non-negative integers $\alpha_{i}, \beta_{j}, 1 \leq i \leq \theta, 1 \leq j \leq \eta$ and $\beta_{1}, \ldots, \beta_{\eta}$ not all zero $\}$.

SteP 3. For every $q \in D$, find the smallest $j_{0} \geq 1$ such that $d<$ $q /\left(k-j_{0}\right)$. Then find some $j=j(q)$ with $j_{0} \leq j \leq k-1$ such that $P(q+j d)$ and $P(q-(k-j) d)$ are $>q_{\theta+\eta}$.

In our application, it is always possible to find $j_{0}$ in Step 3 because $d \leq 22$ and $q \geq q_{\theta+1}^{2} \geq 25$. Also $q-(k-j) d$ is positive since $d<q /\left(k-j_{0}\right)<$ 
$q /(k-j)$ as $j \geq j_{0}$. We derive from the above Algorithm the following result.

Lemma 9. Let $d, k$ and $\mu>0$ be given such that $n+(k-1) d<k^{2} d^{2} / \mu$. If (9) and Step 3 hold, then (8) does not hold.

Proof. Since $n+(k-1) d<k^{2} d^{2} / \mu$, every term $n+i d$ for $0 \leq i \leq k-1$ is of the form $q \in D$ or $q$ with $P(q) \leq q_{\theta}$. Now we follow the proof of [14, Lemma 11] to obtain the assertion of the lemma.

4. Proof of Theorem 2. Let $1<d \leq 22$ and $(n, d, k) \notin\{(2,7,3)$, $(18,7,3),(64,17,3)\}$. We assume that $(9)$ holds and arrive at a contradiction. We apply Lemma 6 to get $k \geq 4$. Next we use Theorem 1 of [14] to derive that $d \geq 7$. Then by Lemma 3 , we may assume that $k \leq k_{0}$ where $k_{0}$ is as given in Table 1 .

Suppose $n \geq(k-1)^{2} d^{2} / 4$. Then we take $\varrho=1$ in Lemma 1 and observe that $\delta(d)=0$. Thus from Lemma 1 , we derive that $t^{\prime}=k$ and hence it follows from Lemma 7 that (9) does not hold. Thus our supposition $n \geq$ $(k-1)^{2} d^{2} / 4$ is false. We assume from now onwards that $n<(k-1)^{2} d^{2} / 4$ and hence

$$
n+(k-1) d<\frac{k^{2} d^{2}}{4} \text { for } 4 \leq k \leq k_{0} \text { and } 7 \leq d \leq 22 .
$$

Suppose $k \geq 27$. Then from Table 1 we have $d \geq 9$. Assume that $n \geq$ $(k-1)^{2} d^{2} / 36$. Then we take $\varrho=9$ in Lemma 1 . Thus $h \leq 2$. Suppose $d=9$. Then $V_{1}=\{1,2,4,5,7,8\}, V_{2}=\{1,2\}, \varepsilon_{1}=1, \varepsilon_{2}=1, \delta_{1}=1$ and $\delta_{2}=1$. Hence $\delta(d) \leq 2$. Similarly, for other values of $d$ we find that $\delta(d) \leq 2$. Hence by Lemma $1, t^{\prime} \geq k-2$. We check that $g(k, 3, d) \geq 9$ for $27 \leq k \leq 66$. This contradicts (23). Thus we derive that

$$
\begin{aligned}
& n+(k-1) d<\frac{(k-1)^{2} d^{2}}{36}+(k-1) d<\frac{k^{2} d^{2}}{32} \\
& \quad \text { for } 27 \leq k \leq 66 \text { and } 9 \leq d \leq 22 .
\end{aligned}
$$

Let $k>66$. Then we see from Table 1 that $d \in\{13,17,19,21,22\}$ and the corresponding upper bound for $k$, viz., $k_{0}$ is large. We use the idea in the preceding paragraph in order to get a good upper bound for $n+(k-1) d$ in different ranges of $k$. First we assume that $n+(k-1) d \geq k^{2} d^{2} / \mu$ for some positive integer $\mu$. Then we find a lower bound for $t^{\prime}$, say $t_{0}$, and a range of $k$, say $R_{1} \leq k \leq R_{2}$, in which by (12), we check that $g(k, m, d) \geq 2^{m}+1$ for a suitable choice of $m$. Since this is a contradiction we derive that

$$
n+(k-1) d<\frac{k^{2} d^{2}}{\mu} \quad \text { for } R_{1} \leq k \leq R_{2} .
$$

In Table 2, we tabulate the choice of $\mu$, values of $t_{0}, R_{1}, R_{2}$ and $m$ when $d \in\{13,17,19,21,22\}$. 
Table 2

\begin{tabular}{ccccc}
\hline$d$ & $\mu$ & $t_{0}$ & $R_{1}-R_{2}$ & $m$ \\
\hline 13 & 92 & $k-5$ & $67-119$ & 4 \\
17 & 92 & $k-5$ & $67-119$ & 4 \\
& 135 & $k-7$ & $120-159,160-174$ & 4,5 \\
& 184 & $k-8$ & $175-215,216-233$ & 5,6 \\
& 240 & $k-10$ & $234-254$ & 6 \\
19 & 32 & $k-2$ & $67-80$ & 4 \\
& 132 & $k-6$ & $81-143,144-174$ & 4,5 \\
& 185 & $k-8$ & $175-302$ & 5 \\
21 & 165 & $k-7$ & $67-99$ & 2 \\
22 & 32 & $k-1$ & $67-74$ & 2 \\
\hline
\end{tabular}

For a given $d, k$, we use (28)-(30) with Table 2 and construct the set $D$ mentioned in Step 2 of the Algorithm in Section 3. Next we proceed to check that Step 3 holds for the given $d, k$ and $q \in D$. This would contradict (8) by Lemma 9 . The verification of Step 3 is done as follows. First, we delete from $D$ all the integers $q$ for which both $P(q+j d)$ and $P(q-(k-j) d)$ exceed $q_{\theta+\eta}$ with $j=j_{0}$. We denote the set of remaining integers of $D$ by $D_{1}$. Secondly if $D_{1} \neq \emptyset$, we delete from $D_{1}$ those integers for which both $P(q+j d)$ and $P(q-(k-j) d)$ exceed $q_{\theta+\eta}$ with $j=j_{0}+1$. The remaining set of integers from $D_{1}$ is denoted by $D_{2}$. The above process is continued till we reach $j=k-1$ or until $D_{i}$ becomes an empty set for some integer $i \geq 1$. For the values of $d$ and $k$ under consideration, we find that we need only take $j$ with $j_{0} \leq j \leq \min (k-1,25)$.

There are triples $(\bar{d}, k, q)$ for which the Algorithm fails, i.e., we are unable to find some $j$ with $j_{0} \leq j<k$ such that both $P(q+j d)$ and $P(q-(k-j) d)$ exceed $q_{\theta+\eta}$. In all, we find 207 triples which are not covered by the Algorithm. For each $d$, we give below a few examples of such triples. For a given $d$, we have chosen as examples those triples for which either $k$ or $q$ is maximum among all the triples $(d, k, q):(7,4,25),(8,5,49)$, $(9,12,169),(10,6,49),(11,12,169),(12,4,49),(13,13,1058),(14,5,363)$, $(14,13,361),(15,5,578),(16,7,361),(16,12,169),(17,7,1058),(17,25,961)$, $(18,5,289),(18,9,121),(19,9,1681),(19,25,961),(20,10,867),(20,16,289)$, $(21,15,361),(21,16,289),(22,6,637),(22,16,289)$.

We observe that in all the 207 cases $k$ and $q$ are not very large. For all these triples $(d, k, q)$, we factorize the product $n(n+d) \ldots(n+(k-1) d)$ directly with $n \in\{q, q-d, \ldots, q-(k-1) d\}$ to find a prime exceeding $k$ which divides the product to an odd power. This completes the proof of Theorem 2.

5. Proof of Theorem 3. Suppose that $d \geq 23$ and $k \geq 4 d(\log d)^{2}$ if $d$ is odd and $k \geq(1.3) d(\log d)^{2}$ if $d$ is even. Then $k>2(2 d-7)$. Also 
by equation (2), we may assume that (9) holds. Thus we conclude from Corollary 1 that $t^{\prime} \geq k-\delta(d)$ where $\delta(d)$ is computed with $\varrho=\frac{1}{3} d^{2}$. By Lemma $4, \delta(d) \leq(.52) d \log d$ for $d$ odd, $\delta(d) \leq(.38) d \log d$ for $d$ even and hence $k-\delta(d) \geq 63$. Hence by Corollary 2(i), we have

$$
\prod_{i=1}^{t^{\prime}} a_{i}^{\prime} \geq \prod_{i=1}^{k-\delta(d)} a_{i}^{\prime} \geq \prod_{i=1}^{k-\delta(d)} s_{i} \geq(1.5)^{k-\delta(d)}(k-\delta(d)) !
$$

We use (31), (21) and (22) to get

$$
1.5549 \leq(153819970)^{1 / k}(1.5)^{\delta(d) / k} k^{(15+\delta(d)) / k} .
$$

Let $d$ be even. Then we turn to sharpening (32). Since $\operatorname{gcd}\left(a_{i}^{\prime}, d\right)=1$ for $1 \leq i \leq t^{\prime}$ we find that $a_{1}^{\prime}, \ldots, a_{t^{\prime}}^{\prime}$ are odd. By Corollary 2(iii), we have

$$
\prod_{i=1}^{t^{\prime}} a_{i}^{\prime} \geq\left(\frac{9}{4}\right)^{k-\delta(d)}(k-\delta(d)) !
$$

We note that $g_{2}=0$ and we use (20) with (19) for $q \geq 3, g_{2}-h_{2} \leq-k+\frac{\log k}{\log 2}$ to estimate

$$
\prod_{i=1}^{t^{\prime}} a_{i}^{\prime} \leq(38454993)(.7657)^{k} k^{15}(k-1) ! .
$$

Finally, we combine the upper and lower estimates for $\prod_{i=1}^{t^{\prime}} a_{i}^{\prime}$ to conclude that

$$
2.9384 \leq(38454993)^{1 / k}\left(\frac{9}{4}\right)^{\delta(d) / k} k^{(14+\delta(d)) / k} \quad \text { for } d \text { even. }
$$

We observe that the right hand sides of the inequalities (32) and (33) are decreasing functions of $k$. Therefore, we put $k=(3.8) d(\log d)^{2}$ in (32) when $d$ is odd and $k=(1.3) d(\log d)^{2}$ in (33) when $d$ is even. By Lemma 4 , we may replace $\delta(d)$ in (32) by $\frac{1}{4} d \log d+(.8323) d$ and $\delta(d)$ in (33) by $\frac{1}{3} d \log d+(.118) d$. The resulting inequalities do not hold for $d \geq 23$.

\section{Proofs of Theorems 4 and 5}

Proof of Theorem 4. We choose

$$
(n, d, y)= \begin{cases}\left(2, x_{0}^{2}-2,2 x_{0} y_{0}\right) & \text { where } x_{0}^{2}-2 y_{0}^{2}=1 \text { with } x_{0} \text { odd, } \\ \left(2, x_{0}^{2}-2,2 x_{0} y_{0}\right) & \text { where } x_{0}^{2}-3 y_{0}^{2}=1 \text { with } x_{0} \text { odd, } \\ \left(1, x_{0}^{2} / 2-1, x_{0} y_{0} / 2\right) & \text { where } x_{0}^{2}-3 y_{0}^{2}=1 \text { with } x_{0} \text { even. }\end{cases}
$$

Then we observe that $(n, d, y)$ is a solution of equation (6) with $B \in\{2,3,6\}$. Now by Lemma $8(\mathrm{i})$, there are infinitely many such triples $(n, d, y)$ satisfying equation (6) with $B \in\{2,3,6\}$. This proves Theorem 4 . 
Proof of Theorem 5. Let $n \notin\{1,2,48\}$. By the remarks following Theorem 5 in Section 1, we need to consider equation (7) with $B=2,3$. Thus we may assume that (9) holds and we shall arrive at a contradiction. We apply Lemma 5 to assume that $\left(a_{0}, a_{1}, a_{2}\right)=(1,1,2)$ if $B=2$ and $\left(a_{0}, a_{1}, a_{2}\right)=(6,1,2)$ if $B=3$. The first case implies $x_{1}^{2}-x_{0}^{2}=1$, which is not possible. In the second case we have $3 x_{0}^{2}+x_{2}^{2}=x_{1}^{2}$ with $x_{0}$ even, and $x_{0}, x_{1}, x_{2}$ pairwise coprime. Hence by Lemma 8 (ii), we have $\varrho_{0}=2$ and $1=x_{1}^{2}-6 x_{0}^{2}=\alpha^{2} u^{4}+\beta^{2} s^{4}-18 u^{2} s^{2}$ with $\alpha \beta=3$. It is no loss of generality to assume that $\alpha=1, \beta=3$ while dealing with this equation. Thus we consider $u^{4}+9 s^{4}-18 u^{2} s^{2}=1$. This implies that $1+8 u^{4}=c_{1}^{2}$ for some integer $c_{1}$. This immediately reduces to the equation $u_{1}^{4}-2 u_{2}^{4}= \pm 1$ for some integers $u_{1}$ and $u_{2}$ with $u_{1} u_{2}= \pm u$. We apply Lemma 8(iii) to observe that $\left(u_{1}, u_{2}\right)=( \pm 1,0)$ or $( \pm 1, \pm 1)$. Hence $u=0$ or \pm 1 . Thus we have $9 s^{4}=1$ or $s^{2}\left(9 s^{2}-18\right)=0$. The former is impossible while the latter gives $s=0$, implying that $x_{0}=0$ by Lemma 8 (ii) and this is not possible.

\section{References}

[1] T. M. Apostol, Introduction to Analytic Number Theory, Springer, 1976.

[2] L. E. Dickson, Introduction to the Theory of Numbers, Univ. of Chicago Press, 1946.

[3] - History of the Theory of Numbers, Vol. II, Washington, 1919; reprint: Chelsea, New York, 1971.

[4] P. Erdős, Note on products of consecutive integers, J. London Math. Soc. 14 (1939), 194-198.

[5] P. Erdős and J. L. Selfridge, The product of consecutive integers is never a power, Illinois J. Math. 19 (1975), 292-301.

[6] W. Ljunggren, New solution of a problem proposed by E. Lucas, Norsk Mat. Tidsskrift 34 (1952), 65-72.

[7] R. Marszałek, On the product of consecutive elements of an arithmetic progression, Monatsh. Math. 100 (1985), 215-222.

[8] A. Meyl, Question 1194, Nouv. Ann. Math. (2) 17 (1878), 464-467.

[9] L. J. Mordell, Diophantine Equations, Academic Press, 1969.

[10] I. Niven and H. S. Zuckerman, An Introduction to the Theory of Numbers, Wiley, 1972.

[11] P. Ribenboim, Catalan's Conjecture, Academic Press, 1994.

[12] O. Rigge, Über ein diophantisches Problem, in: 9th Congress Math. Scand., Helsingfors, 1938, Mercator, 155-160.

[13] J. B. Rosser and L. Schoenfeld, Approximate formulas for some functions of prime numbers, Illinois J. Math. 6 (1962), 64-94.

[14] N. Saradha, On perfect powers in products with terms from arithmetic progressions, Acta Arith. 82 (1997), 147-172.

[15] T. N. Shorey and R. Tijdeman, On the greatest prime factor of an arithmetical progression, in: A Tribute to Paul Erdős, A. Baker, B. Bollobás and A. Hajnal (eds.), Cambridge Univ. Press, 1990, 385-389. 
[16] T. N. Shorey and R. Tijdeman, Perfect powers in products of terms in an arithmetical progression, Compositio Math. 75 (1990), 307-344.

[17] G. N. Watson, The problem of the square pyramid, Messenger Math. 48 (1919), $1-22$.

School of Mathematics

Tata Institute of Fundamental Research

Homi Bhabha Road

Mumbai 400005, India

E-mail: saradha@math.tifr.res.in

Received on 15.7.1997

and in revised form on 16.3.1998 